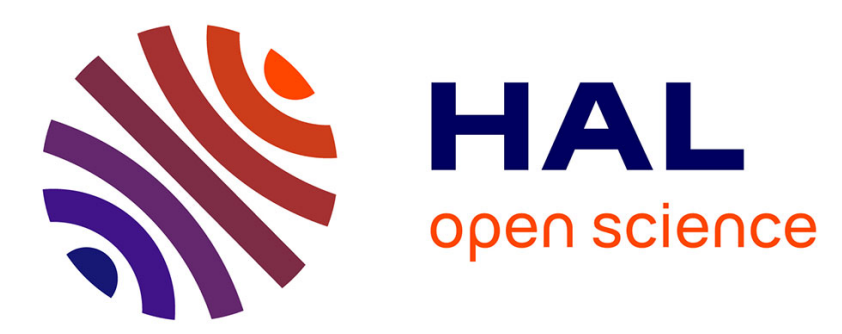

\title{
Ultrasound characterization of aggregated red blood cells : towards in vivo application
}

\author{
Emilie Franceschini, François T. H. Yu, Guy Cloutier
}

\section{To cite this version:}

Emilie Franceschini, François T. H. Yu, Guy Cloutier. Ultrasound characterization of aggregated red blood cells : towards in vivo application. Research Network GDR 2501, Jun 2008, France. pp.293-302. hal-00443787

\section{HAL Id: hal-00443787 \\ https://hal.science/hal-00443787}

Submitted on 4 Jan 2010

HAL is a multi-disciplinary open access archive for the deposit and dissemination of scientific research documents, whether they are published or not. The documents may come from teaching and research institutions in France or abroad, or from public or private research centers.
L'archive ouverte pluridisciplinaire HAL, est destinée au dépôt et à la diffusion de documents scientifiques de niveau recherche, publiés ou non, émanant des établissements d'enseignement et de recherche français ou étrangers, des laboratoires publics ou privés. 
Franceschini et al

\title{
Ultrasound characterization of aggregated red blood cells: towards in vivo application
}

\author{
Emilie Franceschini, François T. H. Yu, Guy Cloutier \\ Laboratory of Biorheology and Medical Ultrasonics \\ University of Montreal Hospital Research Center \\ 2099 Alexandre de Sève (room Y-1619), \\ Montréal, Québec, H2L 2W5, Canada \\ franceschini@1ma.cnrs-mrs.fr
}

\begin{abstract}
Ultrasonic backscattered signals from blood contain frequency-dependent information that can be used to obtain quantitative parameters reflecting the aggregation state of red blood cells (RBCs). The difficulty to use this frequency-dependent information in vivo is due to the attenuation caused by intervening tissue layers that distorts the spectral content of backscattering properties from blood microstructures. We propose an optimization method to simultaneously estimate tissue attenuation and blood structure factor. In an in vitro experiment, we obtained satisfactory estimates with relative errors below $25 \%$ for attenuations between 0.115 and $0.411 \mathrm{~dB} / \mathrm{cm} / \mathrm{MHz}$ and $D<10$ ( $D$ the aggregate diameter expressed in number of RBCs).
\end{abstract}

\section{Introduction}

For the detection and characterization of tissues, quantitative ultrasound techniques using the radio frequency (rf) backscattered signals have received broad interest for the past 30 years. One approach is to use the magnitude and the frequency dependence of the rf backscatter spectrum in order to quantify the tissue structures such as the size, acoustic impedance, and concentration of the scatterers. Many in vitro and in vivo experiments have been performed to demonstrate the utility of this approach for characterizing of eye, liver, kidney, prostate and breast. Recently, the frequency dependence of the ultrasound backscattering coefficient (BSC) was studied to assess the level of red blood cell (RBC) aggregation. Two parameters describing RBC aggregation, the packing factor and mean aggregate diameter, were estimated from the Structure Factor Size Estimator (SFSE). ${ }^{1}$ The difficulty to use the SFSE in vivo is that the spectral content of backscattered signals is also affected by attenuation caused by intervening tissue layers between the probe and the blood flow. More generally, ultrasound scatterer size estimation techniques for tissue characterization (such as liver, kidney, prostate or breast) has had limited success in clinical practice because of tissue attenuation effects. ${ }^{2,3}$ Some groups ${ }^{2,4,5}$ developed measurement methods to evaluate the frequencydependent attenuation in order to compensate a posteriori the backscattered power spectrum. Our goal is to explore an other strategy for in vivo measures of RBC scatterer sizes. Recently, we propose an optimization method providind an estimate of total attenuation and blood structural parameters simultaneously, termed the Structure Factor Size and Attenuation Estimator (SFSAE). ${ }^{6}$ This method consists in fitting the spectrum of the backscattered radio-frequency (rf) echoes from blood to an estimated spectrum by a modified SFSE model. Herein, the SFSAE is improved and in vitro experimental evaluation of the SFSAE is performed. 


\section{Background: Structure Factor Size and Attenuation Estimator}

The technique allowing to estimate simultaneously blood structural parameters and total attenuation has been described in detail in Ref. 6 and is summarized here.

Blood can be considered as a very dense suspension of particles (i.e. red cells) having strong interactions (collision, attraction, deformation, flow dependent motions). We develop a theoretical model of blood ultrasound backscattering based on the particle approach. ${ }^{7,8}$ Thsi approch consists of summing contributions from individual RBCs and models the RBC interaction by a particle paircorrelation function. The model proposed in Ref. 1 has been modified to predict the theoretical backscatter coefficient from blood: ${ }^{6}$

$$
B S C_{\text {theor }}(k)=m \sigma_{b}(k) S(k) A(k)
$$

where $k$ is the wave vector, $m$ is the number density of RBCs in blood estimated by measuring the hematocrit $H$ by microcentrifugation ( $m=H / V_{s}$, where $V_{s}$ is the volume of a RBC), $\sigma_{b}$ is the backscattering cross section of a single RBC, $S$ is the structure factor describing the spatial organization of RBCs, and $A$ is the frequency-dependent attenuation function. The backscattering cross-section $\sigma_{b}$ of a weak scattering particle small compared to the wavelength (Rayleigh scatterer) can be determined analytically as follows: $\sigma_{b}(k)=1 /\left(4 \pi^{2}\right) k^{4} V_{s}^{2} \gamma_{z}^{2}$, where $\gamma_{z}$ is the variation of impedance between the RBC and its suspending medium (i.e. the plasma). The structure factor $S$ is the Fourier transform of the pair-correlation function ${ }^{8} g$ and is approximated by its second-order Taylor expansion ${ }^{1}$ in $k$ as

$$
S(k)=1+m \int(g(r)-1) e^{-2 j k r} d r \approx W-\frac{12}{5}(k R)^{2} .
$$

In this last equation, $W$ is the low-frequency limit of the structure factor $\left(\left.S(k)\right|_{k \rightarrow 0}\right)$ called the packing factor. ${ }^{8,9} R$ is the radius of $3 \mathrm{D} \mathrm{RBC}$ aggregates assumed to be isotropic. We introduce $D=R / a$ as the isotropic diameter of an aggregate (expressed in number of RBCs) with $a$ the radius of one RBC sphere-shaped model of volume $V_{s}$. The attenuation function $A$ is given by: $A(k)=e^{-4 \alpha_{0} f}$, where $f$ is the frequency and $\alpha_{0}$ is the attenuation coefficient (in $\mathrm{dB} / \mathrm{MHz}$ ) defined by: $\alpha_{0}=\sum_{i} \alpha_{i} e_{i}$, where $\alpha_{i}$ and $e_{i}$ are respectively the intervening tissue layer attenuations (in $\mathrm{dB} / \mathrm{cm} / \mathrm{MHz}$ ) and thicknesses. We thus assume that the attenuation increases linearly with the frequency: $\alpha(f)=\alpha_{0} f$.

The measured backscatter coefficient was computed as

$$
B S C_{\text {meas }}(k)=B S C_{\text {ref }}(k) \frac{\overline{P_{\text {meas }}(k)}}{\overline{P_{\text {ref }}(k)}} .
$$

In Eq. (3), the mean backscattered power spectrum $\overline{P_{\text {meas }}}$ was obtained by averaging the power spectra of 20 backscattered echoes from blood. The mean power spectrum $\overline{P_{\text {ref }}}$ was obtained from a reference sample of non-aggregated RBCs at a low hematocrit of $6 \%$ (i.e. Rayleigh scatterers). ${ }^{1} 0$ In this case, 20 echoes were also averaged. This reference sample was used to compensate the backscattered power spectrum $\overline{P_{\text {meas }}}$ for the electromechanical system response, and the depthdependent diffraction and focusing effects caused by the US beam. 
The packing factor $W$, aggregate diameter $D$ and total attenuation along the propagation path $\alpha_{0}$ were determined by matching the measured $B S C_{\text {meas }}$ given by Eq. (3) with the theoretical $B S C_{\text {theor }}$ given by Eq. (1). For this purpose, we searched values of $W, D$ and $\alpha_{0}$ minimizing the cost function $F\left(W, D, \alpha_{0}\right)=\left\|B S C_{\text {meas }}-B S C_{\text {theor }}\right\|^{2}$. In all studied cases, the cost function seemed to have a unique global minimum, as was observed by plotting the cost function surface $F(W, D)$ with varying values of $\alpha_{0}$ (see Fig. 1 in Ref. 6). For the optimization problem, we defined a set of lower and upper bounds on the variables $\left(W, D, \alpha_{0}\right)$ so that the solution is searched in the range: $0 \leq W \leq 100,0 \leq D \leq 50$ and $0 \leq \alpha_{0} \leq 1 \mathrm{~dB} / \mathrm{MHz}$. We chose to reject the solution $(W, D$, $\alpha_{0}$ ) having an estimated diameter $D$ very small compared to 0.1 .

\section{In vitro experiment in a Couette flow device}

\subsection{Blood prepation}

Fresh porcine whole blood was obtained from a local slaughter house, centrifuged and the plasma and buffy coat were removed. Two blood samples were then prepared: (i) a H6 reference sample, which was a $6 \%$ hematocrit non-aggregating RBCs resuspended in saline solution; and (ii) a $40 \%$ hematocrit T40 test sample, which consisted of RBCs resuspended in plasma to promote aggregation.

\subsection{Experimental set up}

US measurements were performed in a Couette flow system to produce a linear blood velocity gradient at a given shear rate. The schematic configuration of the experience is shown in figure 1. The system consisted of a rotating inner cylinder with a diameter of $160 \mathrm{~mm}$ surrounded by a fixed concentric cylinder of diameter $164 \mathrm{~mm}$. A $60 \mathrm{~mL}$ blood sample was sheared in the $2 \mathrm{~mm}$ annular space between the two coaxial cylinders. The US scanner (Vevo 770, Visualsonics, Toronto, Canada) equipped with the RMV 710 probe was used in B-mode. The single-element focused circular transducer had a center frequency of $25 \mathrm{MHz}$, a diameter of $7.1 \mathrm{~mm}$ and a focal depth of 15 $\mathrm{mm}$. We operated at a sampling frequency of $250 \mathrm{MHz}$ with 8 bits resolution (Gagescope, model 8500CS, Montreal, Canada). The probe was mounted in the side wall of the fixed outer cylinder and was positioned to have its focal zone at the center of both cylinders. To ensure ultrasonic coupling, the hole within the outer stationary cylinder (containing the probe) was filled with a liquid agar gel based mixture. When solidified, this gel was cut to match the curvature of the cylinder to avoid any flow disturbance. The gel was a mixture of distilled water, 3\% (w/w) agar powder (A9799, Sigma Chemical, Saint-Louis, MO), 8\% (w/w) glycerol and a specific concentration of 50 $\mu m$ cellulose scattering particles (S5504 Sigmacell, Sigma Chemical, Saint-Louis, MO) that determined the attenuation coefficient. Five experiments were performed with five mixtures having Sigmacell (SC) concentrations varying from $0 \%$ to $1 \%(\mathrm{w} / \mathrm{w})$. Since skin is one of the most attenuating tissue layers during in vivo scanning, phantoms were prepared in order to have attenuations closed from skin attenuation. The $0 \%$ concentration constituted the non-attenuating gel and the four other mixtures mimicked skin attenuations. 


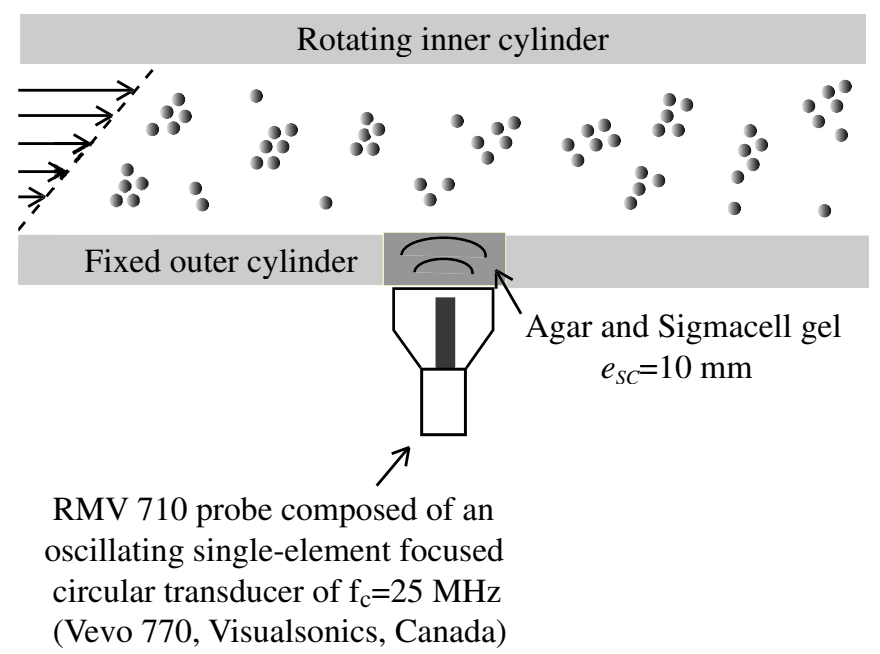

Figure 1: The Couette flow system

\subsection{Attenuation measurements}

The attenuation coefficients of the reference $(0 \%$ SC) and of the others skin-mimicking phantoms $\alpha_{s c}$ were determined by using a standard substitution method. A transducer with center frequency of $25 \mathrm{MHz}$ (Vevo 770, Visualsonics, Toronto, Canada) was used in transmission/reception with a reflector on the opposite side of the phantom for reflection measurements. Reflected signals were recorded both with and without the agar gel sample in the acoustic path. The attenuation coefficient was then estimated using a log spectral difference technique. For each concentration of SC, six regions were scanned for averaging purpose. Thicknesses of skin-mimicking phantoms $e_{s c}$ were fixed to $10 \mathrm{~mm}$. As shown in the table 1 summarizing results, attenuation coefficients of skin-mimicking phantoms were in the same range as the human dermis $(0.21 \mathrm{~dB} / \mathrm{MHz}$ at $14-50$ MHz considering a $1 \mathrm{~mm}$ dermis thickness ${ }^{11}$ ).

Attenuations of the blood $\alpha_{\text {blood }}$ sheared at different shear rates were also measured in the reflection mode using the same experimental configuration shown in figure 1 . The gel had a $0 \%$ $\mathrm{SC}$ concentration and the rotating inner cylinder was used as the reflector. Table 2 summarizes results.

Table 1: Estimated values of the attenuation coefficients of the reference $(0 \% \mathrm{SC})$ and of the others skin-mimicking phantoms (using a log spectral difference technique)

\begin{tabular}{cc}
\hline $\begin{array}{c}\text { SC } \\
(\%)\end{array}$ & $\begin{array}{c}\text { Sigmacell attenuation } \alpha_{s c} \\
(\mathrm{~dB} / \mathrm{MHz})\end{array}$ \\
\hline 0.25 & 0.115 \\
0.5 & 0.219 \\
0.75 & 0.320 \\
1 & 0.412 \\
\hline
\end{tabular}


Table 2: Estimated values of the blood attenuation sheared at different shear rates in the Couette flow device (using a log spectral difference technique)

\begin{tabular}{cc}
\hline $\begin{array}{c}\text { Shear rates } \\
\left(\mathrm{s}^{-1}\right)\end{array}$ & $\begin{array}{c}\text { Blood attenuation } \alpha_{\text {blood }} \\
(\mathrm{dB} / \mathrm{MHz})\end{array}$ \\
\hline 5 & 0.053 \\
10 & 0.036 \\
20 & 0.024 \\
30 & 0.016 \\
50 & 0.013 \\
\hline
\end{tabular}

\subsection{Measurement protocol}

Prior to each measurement, the T40 blood was sheared at $200 \mathrm{~s}^{-1}$ during $30 \mathrm{~s}$ to disrupt RBC aggregates. The shear rate was then reduced to residual values of 5, 10, 20, 30 and $50 \mathrm{~s}^{-1}$ during $90 \mathrm{~s}$ to reach an equilibrium in the state of aggregation in the sheared blood sample. After that, for each shear rate, $20 \mathrm{~B}$-mode images were constructed for $80 \mathrm{~s}$. For each line of the B-mode images, echoes were selected with a rectangular window of length $0.4 \mathrm{~mm}$ at twenty depths every $0.04 \mathrm{~mm}$ (i.e. with $90 \%$ overlap between windows). For each depth, the power spectra of the backscattered echoes were averaged over 20 acquisitions (corresponding to the 20 acquired images) to provide $P_{\text {meas }}$. This protocol was repeated five times with the five agar-based phantoms. Then, the T40 blood was removed and the H6 sample was introduced in the Couette device. The H6 sample was sheared at $50^{-1}$ and coupled with the $0 \%$ SC concentration agar gel. Echoes were windowed as for the $\mathrm{H} 40$ sample at the same depths and their power spectra were averaged over 20 acquisitions to obtain $P_{\text {ref }}$.

\subsection{Reference measurements with the $0 \%$ SC concentration phantom}

The experiment with the $0 \%$ SC phantom was realized in order to have reference results on packing factors $W_{\text {ref }}$ and aggregate diameters $D_{\text {ref }}$ obtained from the classical SFSE. ${ }^{1}$ These parameters were assumed to be true values of packing factors and aggregate diameters for all shear rates, and will be compared in the next section with packing factors and diameters estimated by the SFSAE and by the SFSE when skin-mimicking phantoms are used.

It is important to emphasize the fact that the H6 reference sample was also measured with the $0 \%$ SC phantom. The phantom attenuation, although small with no SC, therefore affected equivalently both spectra $\overline{P_{\text {meas }}}$ and $\overline{P_{\text {ref }}}$ in Eq. (3). The resulting measured backscatter coefficient $B S C_{\text {ref }}$ was thus not biased by attenuation.

\section{Results}

\subsection{Reference parameters with the SFSE}

Figure 2 reports results on $W_{\text {ref }}$ and $D_{\text {ref }}$ from the SFSE with compensation for blood attenuation in the case of no gel attenuation. It can be observed that the amplitude of the backscattering coefficient as well as the estimation of the parameters on $W_{\text {ref }}$ and $D_{\text {ref }}$ decrease when the shear rate increases (i.e. the level of aggregation becomes smaller). 
Franceschini et al

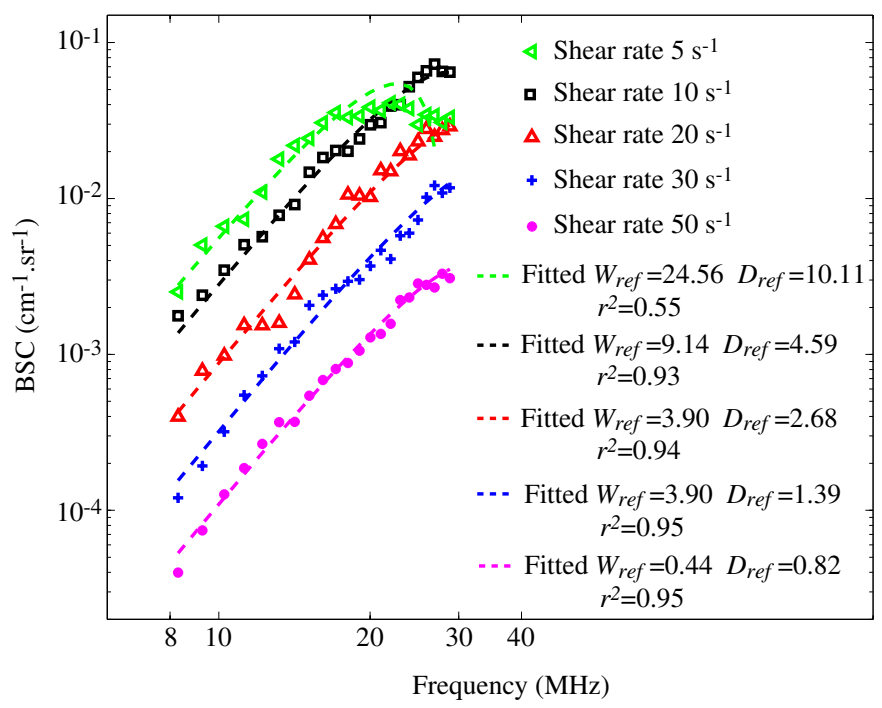

Figure 2: Backscatter coefficients for blood sheared at different residual shear rates and measured with the $0 \%$ SC concentration phantom, and corresponding fitting with the classical SFSE with no compensation for attenuation.
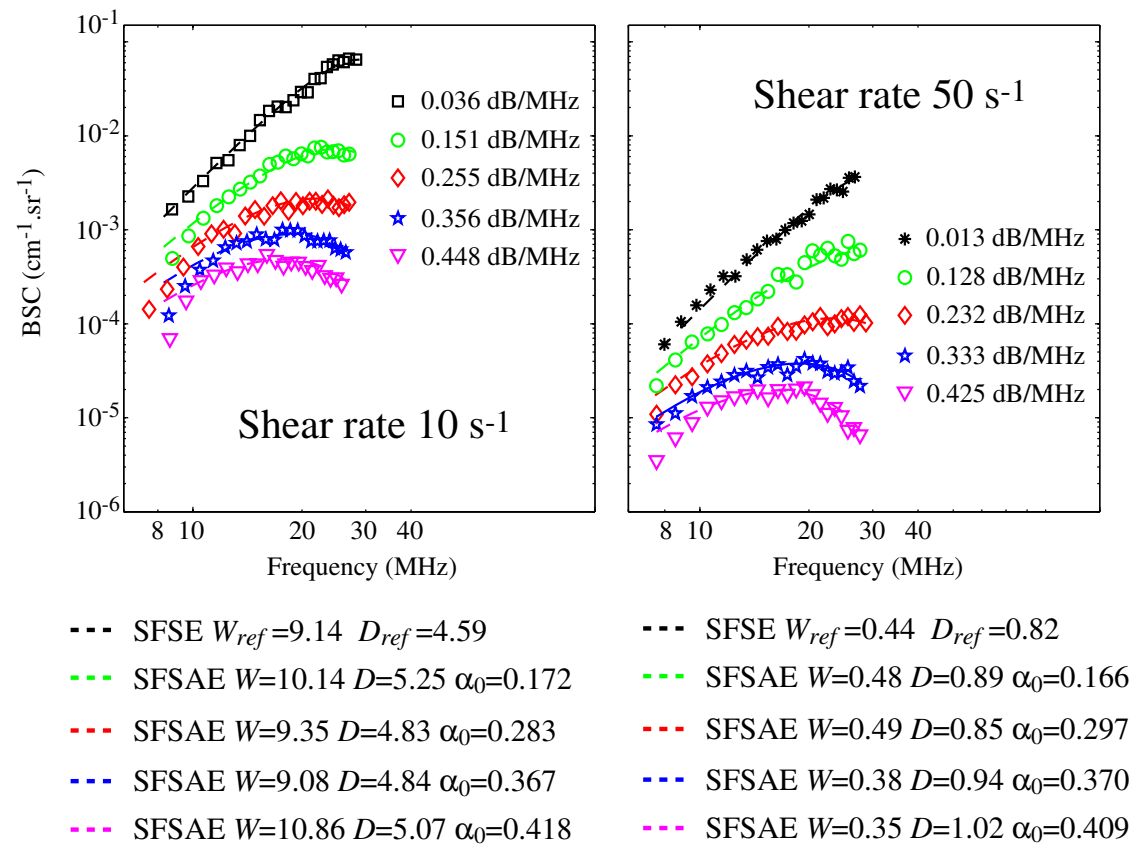

Figure 3: Backscatter coefficients for blood sheared at $10 \mathrm{~s}^{-1}$ and $50 \mathrm{~s}^{-1}$, and measured with each of the five phantoms. The corresponding fitted models are the SFSE for the $0 \%$ SC phantom, and the SFSAE for the four other skin-mimicking phantoms $(0.25,0.5,0.75$ and $1 \%$ SC). 

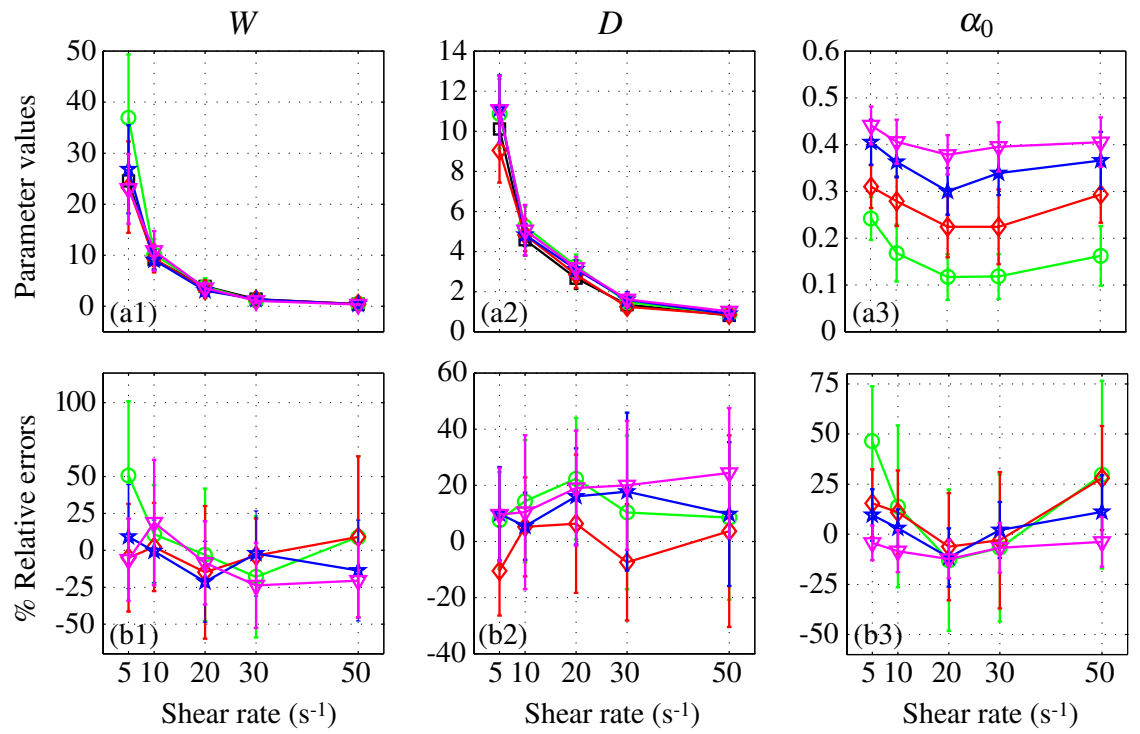

๑ SFSE with compensation for blood attenuation (0\% SC)

๑ SFSAE $(0.25 \%$ SC i.e. $0.115 \mathrm{~dB} / \mathrm{MHz}) \quad$ \ SFSAE $(0.75 \%$ SC i.e. $0.320 \mathrm{~dB} / \mathrm{MHz})$

$\diamond \operatorname{SFSAE}(0.50 \%$ SC i.e. $0.219 \mathrm{~dB} / \mathrm{MHz}) \quad \nabla$ SFSAE $(1 \%$ SC i.e. $0.412 \mathrm{~dB} / \mathrm{MHz})$

Figure 4: (a) Values of $W, D$ and $\alpha_{0}$ (in $\mathrm{dB} / \mathrm{MHz}$ ) for different residual shear rates estimated by the classical SFSE for the $0 \%$ SC concentration and by the SFSAE for the four skin-mimicking phantoms. (b) Corresponding relative errors.
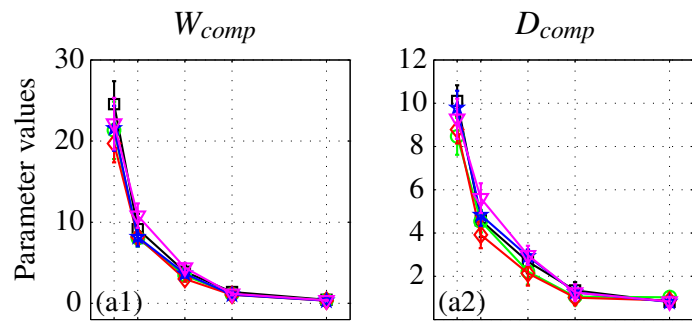

๑ No Sigmacell attenuation
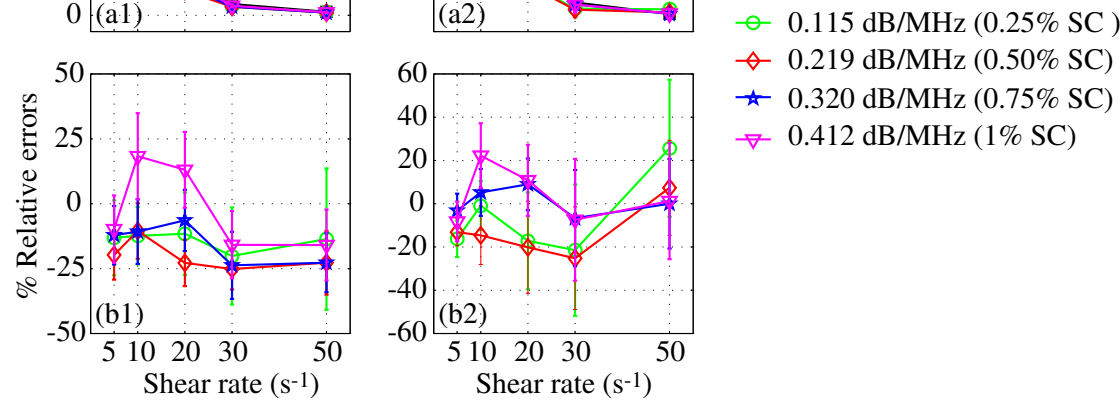

$\dashv 0.219 \mathrm{~dB} / \mathrm{MHz}(0.50 \% \mathrm{SC})$

$\star 0.320 \mathrm{~dB} / \mathrm{MHz}(0.75 \% \mathrm{SC})$

$\rightarrow 0.412 \mathrm{~dB} / \mathrm{MHz}(1 \% \mathrm{SC})$

Figure 5: (a) Values of $W_{\text {comp }}$ and $D_{\text {comp }}$ for the four skin-mimicking phantoms obtained with the SFSE with attenuation-compensation using the attenuation values estimated in reflection. (b) Corresponding relative errors. Parameters $W_{\text {comp }}$ and $D_{\text {comp }}$ are compared with $W_{\text {ref }}$ and $D_{\text {ref }}$. 


\subsection{Parameters evaluated with the SFSAE}

Typical results of the SFSAE minimization procedure for the different agar phantoms at a shear rate of $10 \mathrm{~s}^{-1}$ and $50 \mathrm{~s}^{-1}$ are given in Fig. 3. For both shear rates, it can be observed that more the total attenuation increases, more the backscattering coefficient amplitude decreases at all frequencies and more the frequency dependence of the backscattering coefficient changes. One can also notice that the parameters $W$ and $D$ from the SFSAE are very similar to the reference parameters $W_{\text {ref }}$ and $D_{\text {ref }}$, as well as the total attenuation $\alpha_{0}$ from the SFSAE similar to the reference total attenuation. The reference total attenuation $\alpha_{\text {ref }}$ corresponds to $\alpha_{s c} e_{s c}+\alpha_{\text {blood }} e_{\text {blood }}$ where $\alpha_{s c}$ and $\alpha_{\text {blood }}$ are the skin-mimicking phantom attenuation and the blood attenuation estimated in the reflection mode as shown in section 3.3.

For each residual shear rate, parameters $W, D$ and $\alpha_{0}$ were estimated by the SFSAE. Figure 4 summarizes these results. In this figure, the relative errors for each parameter correspond to: $\left(W-W_{\text {ref }}\right) / W_{\text {ref }},\left(D-D_{\text {ref }}\right) / D_{\text {ref }}$ and $\left(\alpha_{0}-a l p h a_{r e f}\right) / \alpha_{\text {ref }}$. Except for the shear rate $5 \mathrm{~s}^{-1}$ with the skin-mimicking phantom having the smallest attenuation $(0.25 \% \mathrm{SC})$, the SFSAE gave quantitatively satisfactory estimates of $W$ and $D$ with relative errors below $25 \%$.

\subsection{Parameters evaluated with the SFSE with compensation for blood attenuation}

The packing factor $W_{\text {comp }}$ and the diameter of the aggregates $D_{\text {comp }}$ were also evaluated by compensating the backscatter coefficients in the SFSE with the value measured in reflection. Results are presented in Fig. 5. The relative errors are below $25 \%$ for all shear rates and all skinmimicking phantoms.

\section{Conclusions}

The accuracy of the estimates obtained with the SFSAE was as satisfactory as those obtained with the SFSE with attenuation-compensation (i.e when a priori are known about the attenuation). For both methods, relative errors for $W$ and $D$ were below $25 \%$, except for one value corresponding at the shear rate $5 \mathrm{~s}^{-1}$ with the skin-mimicking phantom having the smallest attenuation $(0.25 \%$ SC). In this last case, the SFSAE gave less accurate estimates (relative errors around $50 \%$ for $W$ and $\alpha_{0}$ ). The SFSAE seems to reach its limit of applicability for large aggregate sizes: typically $D_{\text {ref }}=10.11$ (i.e. $k R=2.8$ )

Nevertheless, the SFSAE has the major advantage to be easily applicable in vivo because of the simultaneous estimation of the blood structural properties and total attenuation (contrary to the SFSE attenuation-compensation method, needing the attenuation and thickness of the tissue intervening layers to be known).

\section{Acknowledgments}

This work was supported by the Canadian Institutes of Health Research (grants $\sharp$ MOP-84358 and CMI-72323), by the Heart and Stroke Foundation of Canada (grant $\sharp P G-05-0313$ ), and by the National Institutes of Health of USA (grant $\sharp$ RO1HL078655). Dr Cloutier is recipient of a National Scientist award of the Fonds de la Recherche en Santé du Québec. We are also thankful to Dr F. Destrempes for his helpful discussion on the optimization tool. 


\section{References and links}

${ }^{1}$ F. T. H. Yu and G. Cloutier, "Experimental ultrasound characterization of red blood cell aggregation using the structure factor size estimator", J. Acoust. Soc. Am. 122, 645-656 (2007).

${ }^{2}$ V. Roberjot, S. L. Bridal, P. Laugier, and G. Berger, "Absolute backscatter coefficient over a wide range of frequencies in a tissue-mimicking phantom containing two populations of scatterers", IEEE Trans. Ultras., Ferroelect., Freq. Contr. 43, 970-978 (1996).

${ }^{3}$ T. A. Bigelow, M. L. Oelze, and W. D. O'Brien, "Estimation of total attenuation and scatterer size from backscatter ultrasound waveforms", J. Acoust. Soc. Am. 117, 1431-1439 (2005).

${ }^{4} \mathrm{P}$. He and J. F. Greenleaf, "Application of stochastic analysis to ultrasonic echoes - Estimation of attenuation and tissue heterogeneity from peaks of echo envelope", J. Acoust. Soc. Am. 79, 526-534 (1986).

${ }^{5}$ B. J. Oosterveld, J. M. Thijssen, P. C. Hartman, R. L. Romijn, and G. J. E. Rosenbusch, “Ultrasound attenuation and texture analysis of diffuse liver disease: methods and preliminary results", Phys. Med. Biol 36, 1039-1064 (1991).

${ }^{6}$ E. Franceschini, Y. T. H. Yu, and G. Cloutier, "Simultaneous estimation of attenuation and structure parameters of aggregated red blood cells from backscatter measurements", J. Acoust. Soc. Am. 123, EL85-91 (2008).

${ }^{7}$ L. Y. L. Mo and R. S. C. Cobbold, "Theoretical models of ultrasonic scattering in blood", in Ultrasonic Scattering in Biological Tissues, edited by K. K. Shung and G. A. Thieme (CRC, Boca Raton, FL, 1993), Chap. 5, pp. 125-170.

${ }^{8} \mathrm{~V}$. Twersky, "Low-frequency scattering by correlated distributions of randomly oriented particles”, J. Acoust. Soc. Am. 81, 1609-1618 (1987).

${ }^{9}$ K. K. Shung, "On the ultrasound scattering from blood as a function of hematocrit", IEEE Trans. Ultras., Ferroelect., Freq. Contr. SU-26, 327-331 (1982).

${ }^{10}$ S. H. Wang and K. K. Shung, "An approach for measuring ultrasonic backscattering from biological tissues with focused transducers", IEEE Trans. Biomed. Eng. 44, 549-554 (1997).

${ }^{11}$ B. I. Raju and M. A. Srinivasan, "High-frequency ultrasonic attenuation and backscatter coefficients of in vivo normal human dermis and subcutaneous fat", Ultrasound in Med. Biol. 27, 1543-1556 (2001). 